\title{
Hip Fracture-Related Mortality among Older Adults in the United States: Analysis of the CDC WONDER Multiple Cause of Death Data, 1999-2013
}

\author{
Carlos H. Orces \\ Department of Medicine, Laredo Medical Center, 1700 East Saunders, Laredo, TX 78041, USA \\ Correspondence should be addressed to Carlos H. Orces; corces07@yahoo.com
}

Received 11 October 2015; Revised 7 February 2016; Accepted 8 February 2016

Academic Editor: Jaume Marrugat

Copyright (C) 2016 Carlos H. Orces. This is an open access article distributed under the Creative Commons Attribution License, which permits unrestricted use, distribution, and reproduction in any medium, provided the original work is properly cited.

\begin{abstract}
Objectives. To examine trends in hip fracture-related mortality among older adults in the United States between 1999 and 2013. Material and Methods. The Wide-Ranging Online Data for Epidemiological Research system was used to identify adults aged 65 years and older with a diagnosis of hip fracture reported in their multiple cause of death record. Joinpoint regression analyses were performed to estimate the average annual percent change in hip fracture-related mortality rates by selected characteristics. Results. A total of 204,254 older decedents listed a diagnosis of hip fracture on their death record. After age adjustment, hip fracture mortality rates decreased by $-2.3 \%$ ( $95 \% \mathrm{CI},-2.7 \%$, and $-1.8 \%)$ in men and $-1.5 \%$ ( $95 \% \mathrm{CI},-1.9 \%$, and $-1.1 \%)$ in women. Similarly, the proportion of in-hospital hip fracture deaths decreased annually by $-2.1 \%$ ( $95 \% \mathrm{CI},-2.6 \%$, and $-1.5 \%)$. Of relevance, the proportion of cardiovascular diseases reported as the underlying cause of death decreased on average by $-4.8 \%$ (95\% CI, $-5.5 \%$, and -4.1\%). Conclusions. Hip fracture-related mortality decreased among older adults in the United States. Downward trends in hip fracture-related mortality were predominantly attributed to decreased deaths among men and during hospitalization. Moreover, improvements in survival of hip fracture patients with greater number of comorbidities may have accounted for the present findings.
\end{abstract}

\section{Introduction}

Hip fractures are associated with increased morbidity, functional decline, and mortality among older adults $[1,2]$. Patients with hip fracture have a 5- to 8-fold increased risk of all-cause mortality during the 3 months after the fracture and almost half of the mortality risk during the first year has been attributed to in-hospital deaths. This excess mortality decreases substantially during the first 2 years after fracture but does not return to the mortality rate seen in age- and sexmatched control subjects even after 10 years of follow-up [35].

Previous studies have consistently demonstrated decreasing hip fracture incidence rates in the United States (USA) since the mid-1990s [6-8]. Moreover, hip fracture 30-, 60-, and 360-day adjusted mortality rates among Medicare beneficiaries decreased in both genders between 1986 and 2004 [8]. However, most of this decline in hip fracture mortality occurred before 1998, with a greater downward trend in rates among men. Subsequently, the decrease in hip fracture mortality rates was attenuated in both genders [8]. A recent analysis of the National Hospital Discharge Survey described downward trends in in-hospital hip fracture mortality among older adults in the USA between 1988 and 2007. The decrease in in-hospital mortality after hip fracture during the study period was attributed to decreased death among adults aged 85 years and older, men, and subjects with greater number of comorbidities [9]. Likewise, investigators using data from the Nationwide Inpatient Sample reported that in-hospital hip fracture mortality decreased among Medicare beneficiaries between 1993 and 2010 [10].

According to the Census Bureau's projections, the number of older adults in the USA is expected to more than double over the next decades, rising from 40.3 million in 2010 to 83.7 million by the year 2050 [11]. These demographic changes alone may increase the number of hip fractures and consequently hip fracture-related deaths among older adults. 
Thus, this study aimed to examine trends in hip fracturerelated mortality rates among persons 65 years or older in the USA between 1999 and 2013.

\section{Materials and Methods}

2.1. Data Source. The Centers for Disease Control and Prevention Wide-Ranging Online Data for Epidemiological Research (WONDER) system was used to generate the number of decedents aged 65 years or older with a diagnosis of femoral neck or trochanteric fracture as a contributory cause of death according to the International Classification of Diseases, 10th revision (ICD-10) S72.0 and S72.1 codes, respectively. Death data available on the WONDER system are based on county-level death certificates for U.S. residents. Each death certificate contains demographic data, a single underlying cause of death (UCD), and up to twenty additional multiple causes of death (MCD) [12]. The UCD of death is defined by the World Health Organization as the "disease or injury which initiated the train of events leading directly to the death or the circumstances of the accident or violence which produced the fatal injury." The UCD is selected from the conditions entered by the physician on the cause of death section of the death certificate. When more than one cause or condition is entered by the physician, the underlying cause is determined by the sequence of conditions on the certificate and associated selection rules and modifications [12].

2.2. Statistical Analysis. The joinpoint regression program software, V.4.2.0.2, was used to identify points where a statistically significant change occurred in the linear slope of rates. The joinpoint regression detects points at which significant changes in the direction and magnitude of trends occur over time, assuming constant variance and uncorrelated errors [13]. The tests of significance use the Monte Carlo Permutation method [14]. The results are presented as the average annual percent change (AAPC) and annual percent change (APC) in rates, with their corresponding $95 \%$ confidence interval $(95 \% \mathrm{CI})$. The AAPC in rates is a summary measure of the trend over a prespecified fixed interval. This measure describes the average annual percent changes over a period of multiple years, which is valid even if the joinpoint model indicates that there were changes in trends during those years. The AAPC is computed as a weighted average of the APC in rates from the joinpoint model, with the weights equal to the length of the APC interval [15].

The proportion of hip fracture-related mortality by place of death (hospital and nursing home) and age-specific (65-74, $75-84$, and $\geq 85$ years) and age-adjusted rates by gender per 100,000 of population were generated using the WONDER system. Subsequently, the AAPC in hip fracture-related mortality rates were examined by age groups, gender, and place of death. Moreover, changes in the proportion of selected ICD-10 codes reported as the UCD among hip fracture decedents were examined during the 15 -year study period. In the figures, the observed rates are represented by symbols and the predicted trends from the joinpoint regression model are represented by dashed lines.

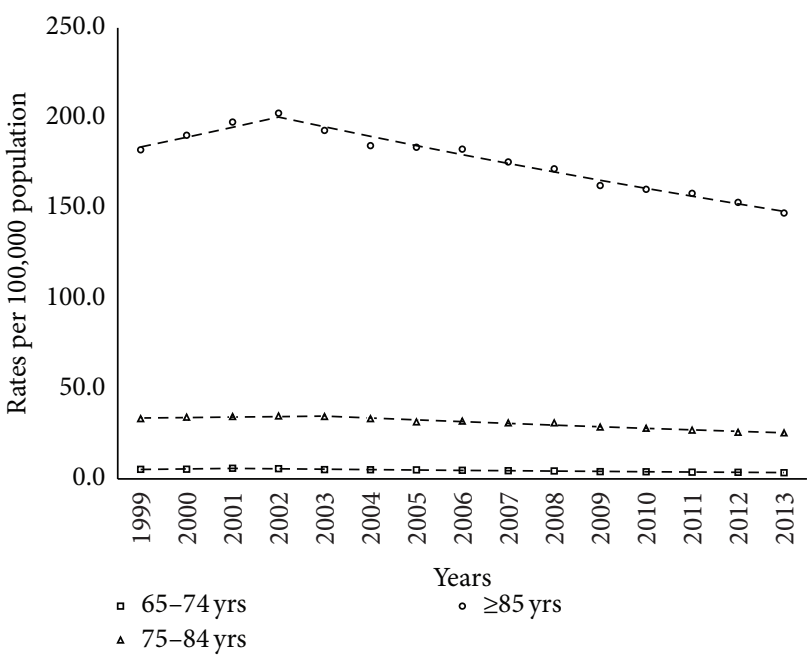

FIGURE 1: Hip fracture-related mortality rates in the USA.

\section{Results}

A total of 204,254 decedents aged 65 years or older listed a diagnosis of hip fracture as a contributory cause of death in their death record between 1999 and 2013. The majority of hip fracture-related deaths occurred in the hospital (49.1\%) and nursing homes (33.1\%). Overall, women and race reported as white accounted for $64.0 \%$ and $95.9 \%$ of hip fracture-related deaths, respectively. The leading underlying causes of death reported among hip fracture decedents were unintentional injuries (49.2\%), diseases of the heart (21.0\%), chronic lower respiratory diseases (4.1\%), Alzheimer's disease (3.7\%), neoplasms (3.5\%), and cerebrovascular diseases (3.1\%).

The number of hip fracture-related deaths increased from 12,643 in 1999 to 13,226 in 2013. However, after age adjustment, hip fracture-related mortality rates decreased from $37.0 / 100,000$ (95\% CI, 36.4\%, and 37.6\%) persons in 1999 to $29.0 / 100,000$ (95\% CI, $28.5 \%$, and $29.5 \%$ ) persons in 2013. As shown in Figure 1, hip fracture-related mortality rates decreased on average by $-2.8 \%$ (95\% CI, $-3.6 \%$, and $-1.9 \%)$ in the age group 65-74 years between 1999 and 2013 . The decrease in mortality was less accentuated in the age groups 75-84 years and 85 years or older by $-2.0 \%$ (95\% CI, $-2.5 \%$, and $-1.4 \%$ ) and $-1.5 \%$ (95\% CI, $-1.9 \%$, and $-1.1 \%)$, respectively. Of relevance, a significant downward trend in hip fracture-related mortality rates by $-2.7 \%$ (95\% CI, -3.0 , $-2.4 \%)$ per year occurred among those aged 85 years and older since 2002 onwards.

As shown in Figure 2, hip fracture age-adjusted mortality rates decreased on average by $-2.3 \%$ (95\% CI, $-2.7 \%$, and $-1.8 \%)$ and $-1.5 \%(95 \% \mathrm{CI},-2.0 \%$, and $-0.9 \%)$ in men and women during the 15 -year study period, respectively. Moreover, a marked annual decrease in age-adjusted rates by $-3.4 \%$ (95\% CI, $-3.8 \%$, and $-3.0 \%)$ occurred in men between 2002 and 2013. Similarly, a decrease in mortality rates by $-2.6 \%$ (95\% CI, $-2.9 \%$, and $-2.2 \%)$ annually was seen among women from 2001 onwards.

By place of death, the crude proportion of hip fracturerelated in-hospital deaths gradually decreased by $-2.1 \%$ 
TABLE 1: Average annual percent changes in selected ICD-10 codes reported as the UCD among hip fracture decedents.

\begin{tabular}{|c|c|c|c|c|}
\hline ICD-10 codes & Number & $(\%)$ & AAPC (95\% CI) & $P$ value \\
\hline Infectious diseases (A00-B99) & 2,288 & 1.1 & $1.1(-0.8,3.0)$ & 0.228 \\
\hline Neoplasms (C00-D48) & 7,547 & 3.6 & $-3.2(-4.0,-2.3)$ & $<0.001$ \\
\hline Diseases of the blood (D50-D89) & 509 & 0.2 & $-0.6(-2.8,1.7)$ & 0.598 \\
\hline Endocrine diseases (E00-E88) & 3,706 & 1.8 & $-3.5(-4.5,-2.4)$ & $<0.001$ \\
\hline Mental disorders (F01-F99) & 8,281 & 4.0 & $9.1(7.5,10.7)$ & $<0.001$ \\
\hline Diseases of the nervous system (G00-G98) & 9,469 & 4.6 & $-0.2(-1.2,0.9)$ & 0.747 \\
\hline Cardiovascular diseases (I00-I99) & 51,959 & 25.4 & $-4.8(-5.5,-4.1)$ & $<0.001$ \\
\hline Respiratory diseases (J00-J98) & 12,069 & 5.9 & $-2.4(-3.0,-1.8)$ & $<0.001$ \\
\hline Digestive diseases (K00-K92) & 2,975 & 1.4 & $-3.8(-5.3,-2.2)$ & $<0.001$ \\
\hline Genitourinary diseases (N00-N99) & 3,166 & 1.5 & $-2.5(-3.8,-1.2)$ & $<0.001$ \\
\hline External causes of morbidity and mortality (V01-Y89) & 101,245 & 49.5 & $2.5(2.1,2.9)$ & $<0.001$ \\
\hline Falls* (W00-W19) & 62,287 & 31.9 & $5.7(5.1,6.4)$ & $<0.001$ \\
\hline
\end{tabular}

${ }^{*}$ Total percentage within the system.

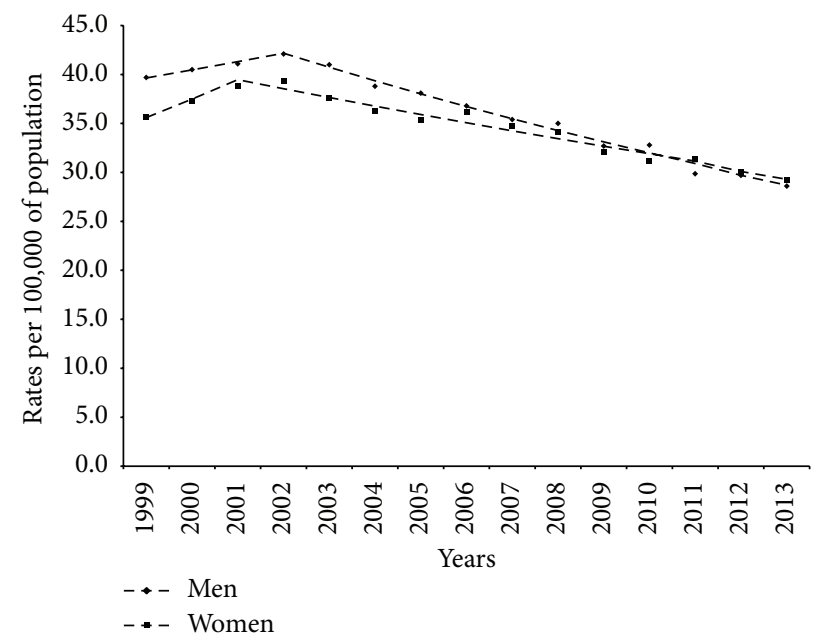

FIGURE 2: Age-adjusted hip fracture-related mortality rates in the USA.

$(-2.6 \%,-1.5 \%)$ per year from $54.9 \%$ in 1999 to $41.5 \%$ in 2013. Likewise, the proportion of hip fracture-related deaths among those residing in nursing homes annually decreased by $-1.4 \%$ ( $95 \% \mathrm{CI},-1.9 \%$, and $-0.8 \%)$. Table 1 shows the distribution and the AAPC in the proportion of selected ICD-10 codes reported as the UCD among hip fracture decedents. Overall, falls represented the leading UCD diagnosis, followed by cardiovascular and respiratory diseases. In addition, trend analyses indicate that cardiovascular diseases reported as the UCD among hip fracture patients significantly decreased by $-4.8 \%$ (95\% CI, $-5.5 \%$, and $-4.1 \%)$ between 1999 and 2013. On the contrary, the report of mental disorders and falls markedly increased on average by $9.1 \%$ (95\% CI, $7.5 \%$, and $10.7 \%)$ and $5.7 \%$ (95\% CI, 5.1\%, and 6.4\%) during the study period, respectively.

\section{Discussion}

The results of the present study indicate that hip fracturerelated mortality significantly decreased among older adults in the USA between 1999 and 2013. These findings were predominantly attributed to marked downward trends in hip fracture-related deaths, among the oldest old, men, and during hospitalization. Consistent with the present findings, a previous study of hip fracture-related mortality in Texas reported a significant decrease in age-adjusted mortality rates among men by $0.8 \%$ annually between 1999 and 2007. On the contrary, a nonsignificant increase in hip fracture-related mortality among women was reported during the study period [16]. Moreover, the significant downward trend in hip fracture-related in-hospital deaths nationwide observed during the 15-year study period is consistent with a recent analysis of the National Hospital Discharge Survey describing decreased hip fracture in-hospital mortality in the USA between 1988 and 2007 [9]. Other studies from France and England have also shown a decrease in hip fracture inhospital deaths in both genders over the past decade $[17,18]$. Possible explanations for the improved survival during hip fracture hospitalization may be related to thromboembolism prophylaxis, management of postoperative complications, and reduced time to surgery [19-22]. Likewise, a randomized clinical trial among subjects aged 50 years and older with a median follow-up time of 1.9 years reported that zoledronic acid administered within 90 days after a hip fracture repair decreased mortality by $28 \%$. However, the authors concluded that further investigation is needed to understand the decreased risk of death associated with the use of this bisphosphonate [23].

Previous studies have documented increased risk of in-hospital and one-year mortality among men after hip fractures $[8,9,16,24]$. Of interest, the present results indicate that hip fracture-related mortality among men has decreased 
since 2002 onwards, which resulted for the first time in lower hip fracture-related mortality rates among men in the USA in 2011. The excess of mortality after hip fractures among men has been attributed to a higher risk of postoperative complications such as pneumonia, delirium, cardiac arrhythmias, and pulmonary embolism [25]. A cohort study using the Clinical Practice Research Datalink from the United Kingdom (UK) reported a decrease in one-year hip fracture mortality for both genders between 2000 and 2010. Moreover, the study described a more pronounced decline in hip fracture mortality among men in the UK [26].

Among hip fracture decedents, the proportion of cardiovascular and respiratory conditions reported as the UCD significantly decreased nationwide by $4.8 \%$ and $2.4 \%$ between 1999 and 2013, respectively. On the contrary, the report of falls and mental conditions markedly increased during the study period. Previous studies have consistently reported that cardiovascular and respiratory diseases are the most common conditions reported among hip fracture decedents $[4,27]$. In fact, a recent analysis of the National Trauma Data Bank demonstrated that in-hospital mortality among hip fracture patients with a cardiac disease was 2.9 times more likely to occur than among those without it [28]. Moreover, cardiac arrhythmia, congestive heart failure, acute myocardial infarction, and chronic obstructive pulmonary disease were the leading listed secondary diagnoses among hip fracture patients who died in the hospital in the USA [9]. Of relevance, hip fracture in-hospital mortality nationwide decreased among older adults with a Charlson comorbidity index score $\geq 2$ from $9.5 \%$ in $1988-1992$ to $5.2 \%$ in 2003 2007 , indicating improvements in the survival of hip fracture patients with greater number of comorbidities, which may also have accounted for the present study results [9]. Although hip fracture in-hospital case fatality rate nationwide has been reported to be $4.9 \%$ in men and $2.6 \%$ in women, the majority of hip fracture-related deaths reported in the death records occurred in the hospital representing about $50 \%$ of decedents [9].

Hip fracture patients with cardiac and respiratory conditions also had 2- and 2.5-fold higher odds of developing major postoperative complications [28]. Despite these facts, a recent study described that cardiovascular and respiratory diseases mortality has decreased among hip fracture patients in the UK over the past decade, which is consistent with the present study results [26]. The marked increase in the report of falls as the UCD among hip fracture decedents may be partially explained by increasing reports of fall as the UCD among older adults with injuries in the USA over the past decade [29].

The present study has several limitations associated with the accuracy of death records in the documentation of hip fractures. First, death certificate data are not validated and some misclassification may occur. Second, hip fractures are generally underreported in the death record. For instance, a previous study demonstrated that, among 2,631 older adults hospitalized with hip fractures during a 7-year period, a total of 930 deaths occurred. Of those decedents, hip fracture was reported in only $5.5 \%$ of the death records [30]. Third, most of the hip fracture-related deaths are accidental and should be reported to the coroner who may request an autopsy or investigation. Therefore, the report of hip fractures is frequently omitted from the death certificate [29]. Fourth, the validation of ICD-10 diagnostic codes in the accuracy of hip fracture-related mortality is undetermined.

In conclusion, hip fracture-related mortality markedly decreased among older adults in the USA between 1999 and 2013. Overall, downward trends in hip fracture-related mortality rates were predominantly attributed to decreased deaths among the oldest old, men, and during hospitalization. Moreover, improvements in survival of hip fracture patients with greater number of comorbidities may have accounted for the present findings.

\section{Conflict of Interests}

The author declares no conflict of interests while performing the present study.

\section{References}

[1] K. W. Lyles, C. S. Colón-Emeric, J. S. Magaziner et al., "Zoledronic acid and clinical fractures and mortality after hip fracture," The New England Journal of Medicine, vol. 357, no. 18, pp. 17991809, 2007.

[2] A. N. A. Tosteson, D. J. Gottlieb, D. C. Radley, E. S. Fisher, and L. J. Melton III, "Excess mortality following hip fracture: the role of underlying health status," Osteoporosis International, vol. 18, no. 11, pp. 1463-1472, 2007.

[3] P. Haentjens, J. Magaziner, C. S. Colón-Emeric et al., "Metaanalysis: excess mortality after hip fracture among older women and men," Annals of Internal Medicine, vol. 152, no. 6, pp. 380390, 2010.

[4] E. S. LeBlanc, T. A. Hillier, K. L. Pedula et al., "Hip fracture and increased short-term but not long-term mortality in healthy older women," Archives of Internal Medicine, vol. 171, no. 20, pp. 1831-1837, 2011.

[5] S. A. Frost, N. D. Nguyen, D. A. Black, J. A. Eisman, and T. V. Nguyen, "Risk factors for in-hospital post-hip fracture mortality," Bone, vol. 49, no. 3, pp. 553-558, 2011.

[6] J. A. Stevens and R. A. Rudd, "The impact of decreasing U.S. hip fracture rates on future hip fracture estimates," Osteoporosis International, vol. 24, no. 10, pp. 2725-2728, 2013.

[7] J. A. Stevens and R. A. Rudd, "Declining hip fracture rates in the United States," Age and Ageing, vol. 39, no. 4, pp. 500-503, 2010.

[8] C. A. Brauer, M. Coca-Perraillon, D. M. Cutler, and A. B. Rosen, "Incidence and mortality of hip fractures in the United States," The Journal of the American Medical Association, vol. 302, no. 14, pp. 1573-1579, 2009.

[9] C. H. Orces, "In-hospital hip fracture mortality trends in older adults: the National Hospital Discharge Survey, 1988-2007," Journal of the American Geriatrics Society, vol. 61, no. 12, pp. 2248-2249, 2013.

[10] M. P. Sullivan, K. D. Baldwin, D. J. Donegan, S. Mehta, and J. Ahn, "Geriatric fractures about the hip: divergent patterns in the proximal femur, acetabulum, and pelvis," Orthopedics, vol. 37, no. 3, pp. 151-157, 2014.

[11] August 2015, http://www.census.gov/library/publications/2014/ demo/p23-212.html.

[12] 2015, http://wonder.cdc.gov/wonder/help/mcd.html\#. 
[13] May 2015, http://surveillance.cancer.gov/joinpoint/.

[14] H.-J. Kim, M. P. Fay, E. J. Feuer, and D. N. Midthune, "Permutation tests for joinpoint regression with applications to cancer rates," Statistics in Medicine, vol. 19, no. 3, pp. 335-351, 2000.

[15] L. X. Clegg, B. F. Hankey, R. Tiwari, E. J. Feuer, and B. K. Edwards, "Estimating average annual per cent change in trend analysis," Statistics in Medicine, vol. 28, no. 29, pp. 3670-3682, 2009.

[16] C. H. Orces and A. H. Alamgir, "Trends in hip fracture-related mortality in Texas, 1990-2007," Southern Medical Journal, vol. 104, no. 7, pp. 482-487, 2011.

[17] M. Maravic, P. Taupin, P. Landais, and C. Roux, "Decrease of inpatient mortality for hip fracture in France," Joint Bone Spine, vol. 78, no. 5, pp. 506-509, 2011.

[18] T.-Y. Wu, M.-H. Jen, A. Bottle, C.-K. Liaw, P. Aylin, and A. Majeed, "Admission rates and in-hospital mortality for hip fractures in England 1998 to 2009: time trends study," Journal of Public Health, vol. 33, no. 2, pp. 284-291, 2011.

[19] D. Marsland, S. C. Mears, and S. L. Kates, "Venous thromboembolic prophylaxis for hip fractures," Osteoporosis International, vol. 21, supplement 4, pp. 593-604, 2010.

[20] N. Rosencher, C. Vielpeau, J. Emmerich, F. Fagnani, and C. M. Samama, "Venous thromboembolism and mortality after hip fracture surgery: the ESCORTE study," Journal of Thrombosis and Haemostasis, vol. 3, no. 9, pp. 2006-2014, 2005.

[21] P. Carpintero, J. R. Caeiro, R. Carpintero, A. Morales, S. Silva, and M. Mesa, "Complications of hip fractures: a review," World Journal of Orthopaedics, vol. 5, no. 4, pp. 402-411, 2014.

[22] A. Bottle and P. Aylin, "Mortality associated with delay in operation after hip fracture: observational study," British Medical Journal, vol. 332, no. 7547, pp. 947-950, 2006.

[23] K. W. Lyles, C. S. Colón-Emeric, J. S. Magaziner et al., "Zoledronic acid and clinical fractures and mortality after hip fracture," The New England Journal of Medicine, vol. 357, no. 18, pp. 17991809, 2007.

[24] Y. Endo, G. B. Aharonoff, J. D. Zuckerman, K. A. Egol, and K. J. Koval, "Gender differences in patients with hip fracture: a greater risk of morbidity and mortality in men," Journal of Orthopaedic Trauma, vol. 19, no. 1, pp. 29-35, 2005.

[25] L. E. Wehren, W. G. Hawkes, D. L. Orwig, J. R. Hebel, S. I. Zimmerman, and J. Magaziner, "Gender differences in mortality after hip fracture: the role of infection," Journal of Bone and Mineral Research, vol. 18, no. 12, pp. 2231-2237, 2003.

[26] C. Klop, P. M. J. Welsing, C. Cooper et al., "Mortality in British hip fracture patients, 2000-2010: a population-based retrospective cohort study," Bone, vol. 66, pp. 171-177, 2014.

[27] J. Panula, H. Pihlajamäki, V. M. Mattila et al., "Mortality and cause of death in hip fracture patients aged 65 or older-a population-based study," BMC Musculoskeletal Disorders, vol. 12, article 105, 2011.

[28] P. J. Belmont Jr., E. J. Garcia, D. Romano, J. O. Bader, K. J. Nelson, and A. J. Schoenfeld, "Risk factors for complications and in-hospital mortality following hip fractures: a study using the National Trauma Data Bank," Archives of Orthopaedic and Trauma Surgery, vol. 134, no. 5, pp. 597-604, 2014.

[29] J. A. Stevens and R. A. Rudd, "Circumstances and contributing causes of fall deaths among persons aged 65 and older: United States, 2010," Journal of the American Geriatrics Society, vol. 62, no. 3, pp. 470-475, 2014.

[30] L. J. Donaldson, L. Parsons, and A. J. Cook, "Death certification in fractured neck of femur," Public Health, vol. 103, no. 4, pp. 237-243, 1989. 


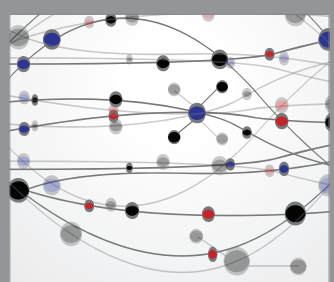

The Scientific World Journal
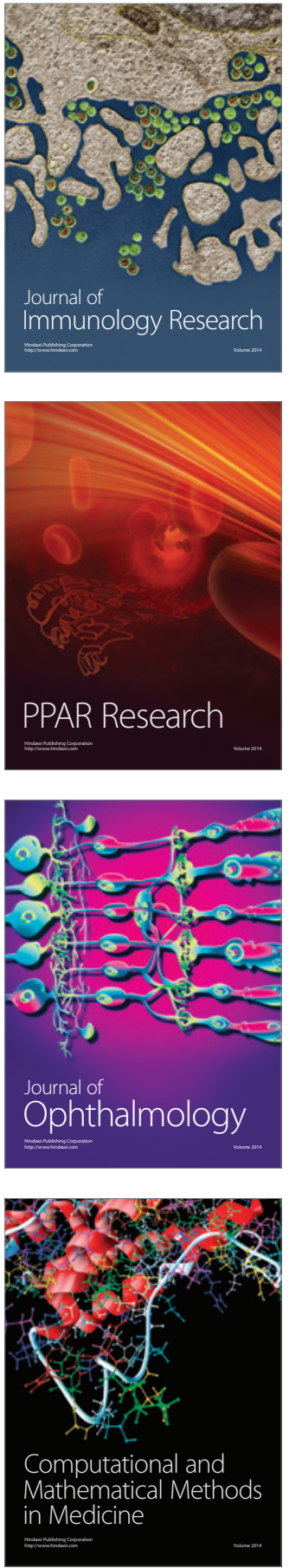

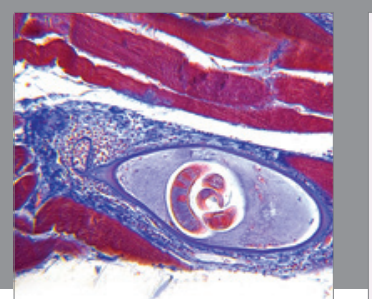

Gastroenterology Research and Practice

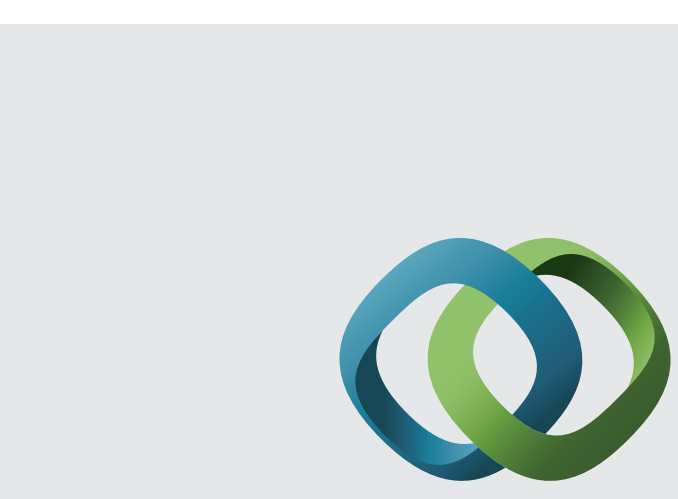

\section{Hindawi}

Submit your manuscripts at

http://www.hindawi.com
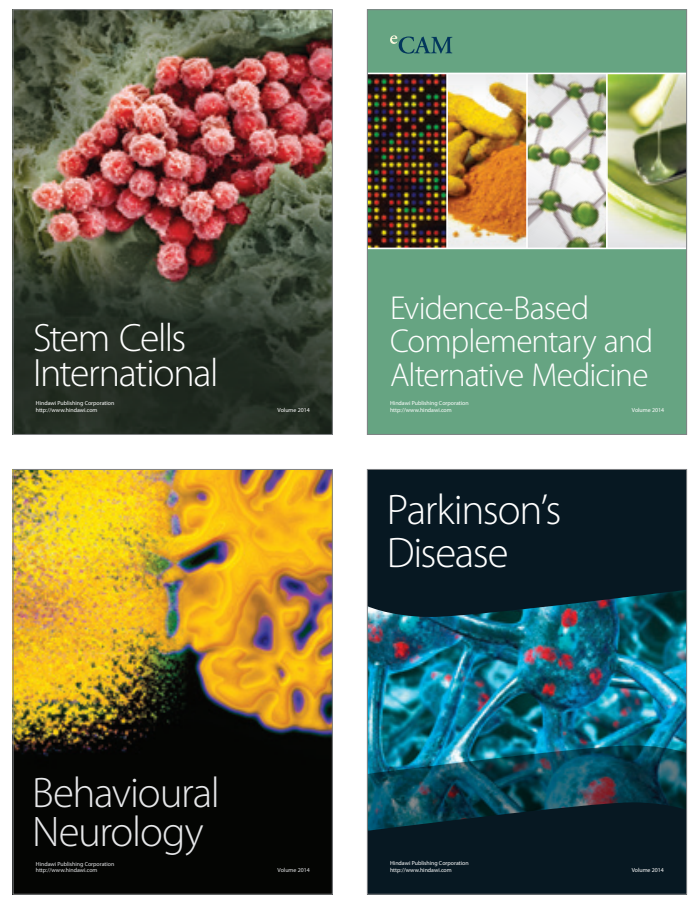
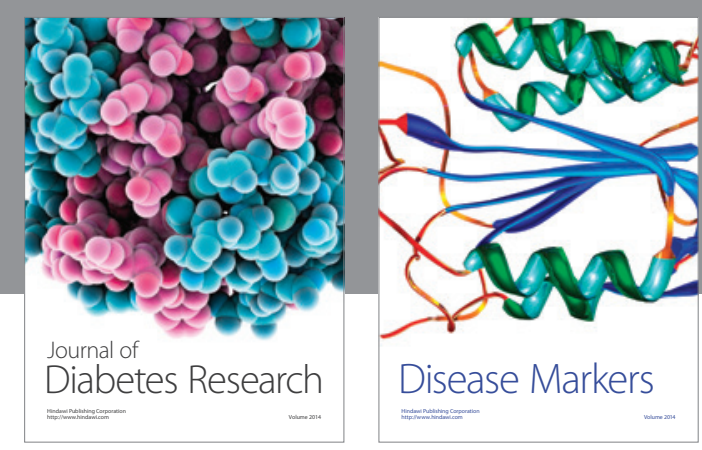

Disease Markers
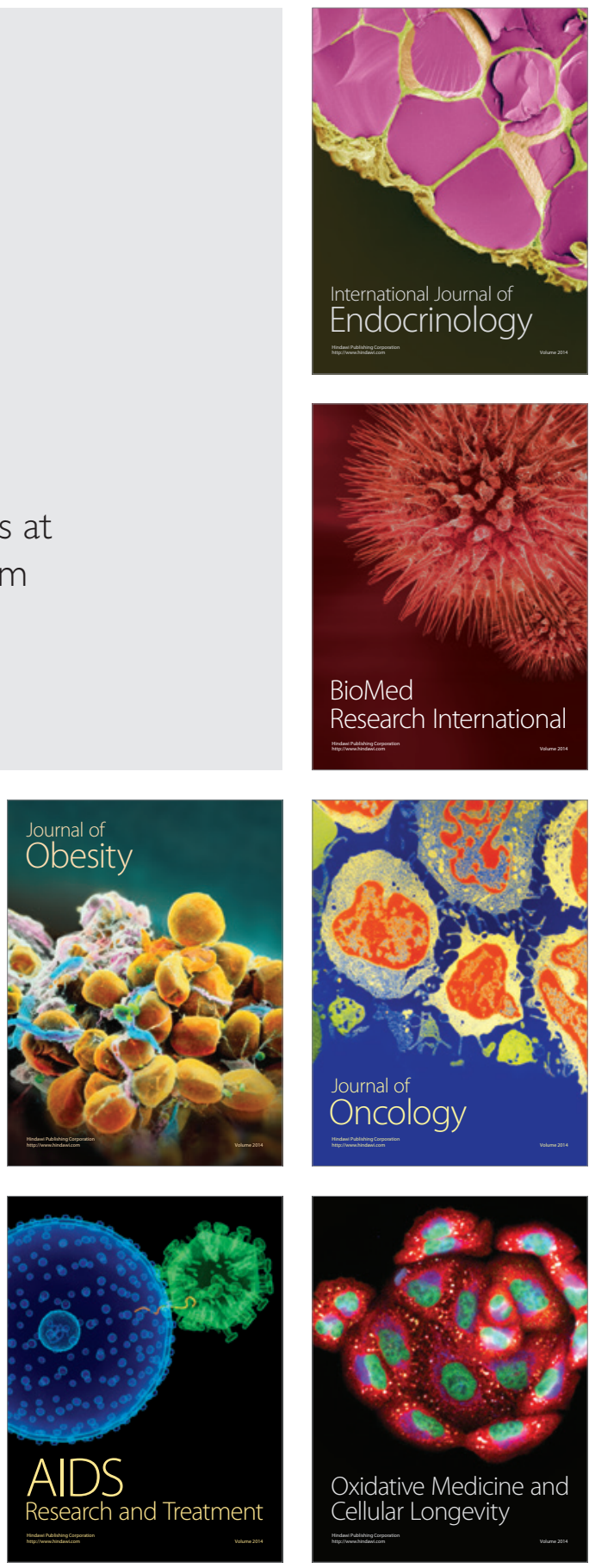